\title{
Characteristics of pathogenic microorganisms found in 99 cases of conjunctivitis from the Qinghai Tibetan area
}

\author{
Benshan Yang, Xiuyuan Li, Qingfeng Liang, Shaoya Zhang \& Shijing Deng* \\ Beijing Institute of Ophthalmology, Beijing Tongren Eye Center, Beijing Tongren Hospital, Capital Medical University, \\ Beijing Key Laboratory of Ophthalmology and Visual Sciences, Beijing 100005, China
}

Received April 1, 2016; accepted April 8, 2016

Citation: Yang, B., Li, X., Liang, Q., Zhang, S., and Deng, S. (2016). Characteristics of pathogenic microorganisms found in 99 cases of conjunctivitis from the Qinghai Tibetan area. Sci China Life Sci 59, 571-572. doi: 10.1007/s11427-016-5061-2

Dear Editor,

Morbidity associated with conjunctivitis is higher in developing countries, particularly among children, because of the poorer standards of living (Azari and Barney, 2013; Yetman and Coody, 1997). Presently, little is known regarding the microorganisms that cause conjunctivitis in the remote area of Chinese Tibet. In particular, it is difficult to monitor and control the occurrence of conjunctivitis in children from this area. Conjunctivitis has two forms: acute and chronic. Its clinical manifestations include conjunctival congestion, edema, photophobia, tearing, itching, and foreign body sensation, and it is predominately caused by bacterial or viral infection. Although conjunctivitis can resolve itself, cases involving infection by Chlamydia trachomatis or pyogenic bacteria can be serious and may threaten vision (Epling, 2012). Currently, China lacks a standardized method for diagnosing infectious ocular disease and the existing do not entirely reflect the etiology of conjunctivitis.

To obtain more relevant information, 99 cases of conjunctivitis were identified by screening Tibetan students from the Jianshetang Primary School and Central Primary School in the Galeng Tibetan Township of Qinghai Xunhua Salar Autonomous County. Screenings were conducted on May 25 and December 31, 2015, respectively. A control cohort of 60 healthy students was evenly and randomly selected from the two schools. Specimens collected were in-

*Corresponding author (email: dengsj@163.com) oculated onto Colombia's blood, chocolate and MacConkey media by using a disposable sterile loop, identified using the Biotyper matrix-assisted laser desorption/ionization time of flight mass spectrometry (MALDI-TOF-MS), and selected for the Kirby-Bauer or minimum inhibitory concentration drug sensitivity test. Chlamydia trachomatis, adenovirus, herpes simplex virus, herpes zoster virus, cytomegalovirus, and Epstein-Barr virus were screened by real-time fluorescent quantitative PCR. Chlamydia trachomatis classification was performed using ompA gene sequencing (Bandea et al., 2001).

Among the 99 cases of conjunctivitis recruited for this study, the total pathogenic microorganism detection rate were 57.58\% (57/99). Across the Jianshetang and Central Primary Schools, the detection rates were $55.81 \%$ (24/43) and $58.93 \%$ (33/56), respectively; the difference between groups was not statistically different. The detection rates in males and females were $43.48 \%(20 / 46)$ and $43.40 \%$ (23/53), respectively, and the results showed no significant bias based on gender. In the control group, pathogenic microorganisms were detected in $8.33 \%$ (5/60) of participants, which is significantly lower than in the conjunctivitis group. The pathogenic microorganism detection rates in the conjunctivitis and control groups are listed in Table 1. In the Jianshetang Primary School, the detection rates of C. trachomatis, Haemophilus influenzae, and Streptococcus pneumoniae were significantly higher in the conjunctivitis group compared to in the control group. In the Central Primary School, the detection rate of $C$. trachomatis in the conjunctivitis group was significantly higher than that in the control group. The detection rate of $S$. pneumoniae in the 
Table 1 Pathogenic microorganism detection rates in the conjunctivitis and control groups

\begin{tabular}{|c|c|c|c|c|c|c|c|c|c|c|c|c|}
\hline \multirow{3}{*}{ pathogen } & \multicolumn{4}{|c|}{ Jianshetang Primary School } & \multicolumn{4}{|c|}{ Central Primary School } & \multicolumn{4}{|c|}{ Total } \\
\hline & \multicolumn{2}{|c|}{$\begin{array}{c}\text { Conjunctivitis group } \\
(n=43)\end{array}$} & \multicolumn{2}{|c|}{$\begin{array}{c}\text { Control group } \\
(n=30)\end{array}$} & \multicolumn{2}{|c|}{$\begin{array}{c}\text { Conjunctivitis group } \\
(n=56)\end{array}$} & \multicolumn{2}{|c|}{$\begin{array}{c}\text { Control group } \\
(n=30)\end{array}$} & \multicolumn{2}{|c|}{$\begin{array}{c}\text { Conjunctivitis group } \\
(n=99) \\
\end{array}$} & \multicolumn{2}{|c|}{$\begin{array}{c}\text { Control group } \\
(n=60)\end{array}$} \\
\hline & Cases & $\begin{array}{l}\text { Detection } \\
\text { rate }(\%)\end{array}$ & Cases & $\begin{array}{c}\text { Detection } \\
\text { rate }(\%)\end{array}$ & Cases & $\begin{array}{c}\text { Detection } \\
\text { rate }(\%)\end{array}$ & Cases & $\begin{array}{c}\text { Detection } \\
\text { rate }(\%)\end{array}$ & Cases & $\begin{array}{c}\text { Detection } \\
\text { rate }(\%)\end{array}$ & Cases & $\begin{array}{c}\text { Detection } \\
\text { rate }(\%)\end{array}$ \\
\hline C. trachomatis & 8 & 18.60 & 0 & 0 & 18 & 32.14 & 0 & 0 & 26 & 26.26 & 0 & 0 \\
\hline H. influenzae & 7 & 16.28 & 0 & 0 & 12 & 21.43 & 2 & 6.67 & 19 & 19.19 & 2 & 3.33 \\
\hline S. pneumoniae & 9 & 20.93 & 0 & 0 & 1 & 1.79 & 0 & 0 & 10 & 10.10 & 0 & 0 \\
\hline M. catarrhalis & 5 & 11.63 & 0 & 0 & 4 & 7.30 & 3 & 10.00 & 9 & 9.09 & 3 & 5.00 \\
\hline S. aureus & 0 & 0 & 0 & 0 & 4 & 7.14 & 0 & 0 & 4 & 4.04 & 0 & 0 \\
\hline N. meningitidis & 1 & 2.33 & 0 & 0 & 0 & 0 & 0 & 0 & 1 & 1.01 & 0 & 0 \\
\hline Adenovirus & 0 & 0 & 0 & 0 & 1 & 1.79 & 0 & 0 & 1 & 1.01 & 0 & 0 \\
\hline
\end{tabular}

Jianshetang Primary School was higher than that in the Central Primary School. In the conjunctivitis group, the detection rate of pathogenic bacteria was $47.47 \%(47 / 99)$ and $C$. trachomatis was $26.26 \%$ (26/99), which were all serotype B, and adenovirus was detected in one case (serotype D). Among the 26 cases with $C$. trachomatis, 14 had a simultaneous infection of another pathogenic bacterium, including eight cases with $H$. influenzae, three cases with Moraxella catarrhalis, two cases with $S$. pneumoniae, and one case with Staphylococcus aureus. For antimicrobial susceptibility test results, $S$. pneumoniae were resistant to erythromycin, clindamycin, and sulfamethoxazoletrimethoprim and showed no $\beta$-lactamase production. The rates of $\beta$-lactamase generation from $S$. aureus and M. catarrhalis were $100 \%(4 / 4)$ and $66.7 \%$ (6/9), respectively, while there was no methicillin-resistant $S$. aureus strain.

In summary, the three pathogens most commonly associated with conjunctivitis were $S$. pneumoniae, $H$. influenzae, and $M$. catarrhalis among students from the Jianshetang Primary School, with the former two significantly more prevalent in cases compared to in controls. In the Central Primary School, the main pathogenic bacteria included $H$. influenzae, $M$. catarrhalis, and S. aureus. The differences in the detection rates of the pathogenic microorganisms between the two schools may be related to several factors, such as location (Frankel et al., 2012), temperature, and humidity (Frankel et al., 2012; West et al., 2015). These findings are similar to those reported by Azari et al. and Narayana and McGee (Azari and Barney, 2013; Narayana and McGee, 2015). The composition of pathogenic microorganisms in conjunctivitis is associated with age and bacteria cause $50 \%-70 \%$ of acute conjunctivitis in children. Haemophilus influenzae, S. pneumoniae, and M. catarrhalis have been reported as the most prevalent bacterial species associated with conjunctivitis in children (Narayana and McGee, 2015; LaMattina and Thompson, 2014). Antibiotic sensitivity tests detected no methicillin-resistant $S$. aureus or $S$. pneumoniae strains producing $\beta$-lactamase. However, all $S$. aureus isolates produced $\beta$-lactamase and all $S$. pneumoniae isolates were resistant to erythromycin, clindamycin, and sulfamethoxazole-trimethoprim. C. trachomatis detected in the conjunctivitis cases were all sero- type B, which is the dominant species in Asia (Takahashi et al., 2007). There was no discrepancy in the detection rates of C. trachomatis between the two schools. In addition, we found that ocular co-infection with $C$. trachomatis and pathogenic bacteria were common, particularly with $H$. influenzae. Therefore, additional consideration should be given to the diagnosis and treatment of trachoma conjunctivitis in this region. In conclusion, our results improve the understanding of pathogenic microorganisms associated with conjunctivitis in China.

Compliance and ethics The author(s) declare that they have no conflict of interest.

Acknowledgements We sincerely appreciate the assistance and support for this study provided by the Qinghai Province People's Government and the Department of Health, Xunhua County Government in Qinghai Province, as well as the Qinghai Province People's Hospital.

Azari A.A., and Barney, N.P. (2013). Conjunctivitis: a systematic review of diagnosis and treatment. JAMA 310, 1721-1729.

Bandea, C.I., Kubota, K., Brown, TM., Kilmarx, P.H., Bhullar, V., Yanpaisarn, S., Chaisilwattana, P., Siriwasin, W., and Black, C.M. (2001). Typing of Chlamydia trachomatis strains from urine samples by amplification and sequencing the major outer membrane protein gene (omp1). Sex Transm Infect 77, 419-422.

Epling, J. Bacterial conjunctivitis. (2012). BMJ Clin Evid pii: 0704.

Frankel, M., Bekö, G., Timm, M., Gustavsen, S., Hansen, E.W., and Madsen, A.M. (2012). Seasonal variations of indoor microbial exposures and theirrelation to temperature, relative humidity, and air exchange rate. Appl Environ Microbiol 78, 8289-8297.

LaMattina, K., and Thompson, L. (2014). Pediatric conjunctivitis. Dis Mon 60, 231-238.

Narayana, S., and McGee, S. (2015). Bedside diagnosis of the "Red Eye": a systematic review. Am J Med 128, 1220-1224.

West, S.K., Munoz, B.E., Mkocha, H., Gaydos, C., and Quinn, T. (2015). Risk of infection with Chlamydia trachomatis from migrants to communities undergoing mass drug administration for trachoma control. Ophthalmic Epidemiol 22, 170-175.

Takahashi, S., Yamazaki, T., Satoh, K., Inoue, M., Takahashi, S., Ishihara, O., Oka, Y., Horiguchi, Y., Okuwaki, Y., Suzuki, S., and Kishimoto, T. (2007). Longitudinal epidemiology of Chlamydia trachomatis serovars in female patients in Japan. Jpn J Infect Dis 60, 374-376.

Yetman, R.J., and Coody, D.K. (1997). Conjunctivitis: a practice guideline. J Pediatr Health Care 11, 238-241.

Open Access This article is distributed under the terms of the Creative Commons Attribution License which permits any use, distribution, and reproduction in any medium, provided the original author(s) and source are credited. 